\title{
Presentation and Management of HHV6 Infection in a 32 Days Old Baby
}

\author{
El Ahmar Malak ${ }^{1}$, Srour Sara ${ }^{1}$, Hanna Pielly ${ }^{1}$, Beih Farah ${ }^{1}$, Sacy Robert ${ }^{2 *}$ and Ibrahim Nada ${ }^{3}$ \\ ${ }^{1}$ Department of Pediatrics, Lebanese University, Lebanon \\ ${ }^{2}$ Department of Pediatrics, Balamand University, Lebanon \\ ${ }^{3}$ Department of Pediatrics, Lebanon
}

*Corresponding author: Sacy Robert, Department of Pediatrics, Balamand University, Lebanon

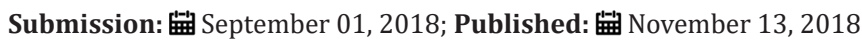

\begin{abstract}
Background: Human herpesvirus 6 (HHV-6), commonly known to cause the childhood disease roseola and nonspecific viral illness, has been known to cause serious neurological complications including fever induced seizures and encephalitis with possible long-term sequelae especially in immunocompromised patients.
\end{abstract}

Purposes: To report a rare case of HHV-6 meningitis in a 32-day old male infant and the challenging therapeutic approach required to treat and manage this condition.

Case summary: A 32-day old male infant presented with one episode of high-grade fever and bulging anterior fontanel in the absence of skin rashes. Blood tests were normal but lumbar puncture done at admission revealed lymphocytic pleocytosis secondary to acute HHV-6 confirmed by CSF PCR. Data indicating appropriate treatment in this age group is limited. In this case, the patient received Ganciclovir IV for 14 days and showed clinical improvement.

Conclusion: Treatment of HHV-6 meningitis with ganciclovir is recommended for immunocompromised patients, including immune deficient patients, and those receiving chemotherapy or prolonged course of steroid. However, literature does not support any specific treatment for immunocompetent children. No data or clear recommendations are currently available for the treatment of neonates with HHV-6 meningitis. Should neonates be thought of as immunocompromised hosts and be treated with Ganciclovir or should they only receive supportive treatment? More studies are required to have an evidence based antiviral treatment in this age group.

Keywords: HHV-6; Meningitis; Ganciclovir

Abbreviation: HHV-6: Human Herpes Simplex-6

\section{Introduction}

HHV-6 is the sixth member of the beta herpes subfamily and is a DNA virus that is expressed as two closely related variants, $\mathrm{A}$ and B [1]. Among the human herpesviruses HHV6 and 7 are most closely related to Cytomegalovirus (CMV). Only 20\% of infected children will develop roseola infantum and the other $80 \%$ will have undifferentiated febrile illness without rash or localized signs. HHV-6B is more common and frequently demonstrated to be the causative agent of roseola infantum in healthy infants [2]. By the age of 3 years, most of children have contracted the infection and manifested as a nonspecific febrile illness [1]. However, HHV6 infection may be complicated by seizure, and more serious central nervous system complications, likely due to its neurotropic properties, including encephalopathy, aseptic meningitis, meningoencephalitis, hemiplegia and multiple sclerosis and other long-term neurological sequelae $[3,4]$.

\section{Case Report}

32-day old male infant presented to our emergency department for his first episode of high-grade fever reaching 39 degrees Celsius associated with one day history of cough. The mother noted good activity and adequate PO intake. There were no associated gastrointestinal symptoms (i.e., diarrhea or vomiting), no irritability, no abnormal movements, no skin rash (petechia purpura), no mottled skin, no hypoactivity, and no cyanosis. The infant was born full tern by normal vaginal delivery, no neonatal intensive care unit (NICU) admission, previously healthy, to a 25 years old healthy mother, moderate smoker, well followed pregnancy, smooth course, non-complicated, GBS and TORCH status not available. G6P3A3: The mother had three previous abortions all in the first trimester of pregnancy without obvious cause. There is no consanguinity between the parents. 
Currently the infant is taking a vitamin D (400IU) supplementation but no other medications. He is breastfed, and bottle fed with regular cow's milk. He received his first vaccination at birth (hepatitis B). The baby also has two healthy siblings, a 3yo sister and 1.5yo brother, with a recent history of isolated fever without skin rash or headache. Physical exam: Upon arrival to the emergency department, the baby was active, tonic, playful, had fever, 38.9 degrees Celsius, other vital signs were normal. The physical exam was unremarkable except for an appreciable bulging anterior fontanelle. No visible skin rash or irritability were noted. Laboratory: Immediate full sepsis workup was done, starting with complete blood test, urine analysis, stool analysis, chest X-ray, lumbar puncture and cultures. Blood tests were unremarkable with WBC of 9000 and neutrophils at $24 \%$ and negative C-reactive protein (CRP). Examination of his cerebrospinal fluid performed on the day of presentation revealed CSF-WBC 265 and RBC 52 with $82 \%$ lymphocytic and $18 \%$ segmented. CSF-protein was $1.04 \mathrm{~g} / \mathrm{L}$ and CSF-glucose was $32 \mathrm{mg} / \mathrm{dL}$; both within normal range. Urine analysis was negative, stool analysis revealed neither adenovirus nor rotavirus. Chest X-ray showed no abnormalities. Five days after initial admission to the hospital, urine, stool, blood culture, and CSF showed no growth of any pathogens. CSF sent for real time polymerase chain reaction (PCR) using the multiplex PCR to detect multiple viral pathogens. PCR for HHV-6 in CSF was positive. However, PCR for all other viral pathogens were negative including: Epstein-Barr virus (EBV), cytomegalovirus (CMV), adenovirus, herpes simplex viruses 1 and 2 (HSV1 and 2), varicella zoster virus (VZV), human herpes virus 7 (HHV7), and parvovirus. During his stay in the hospital, the infant was hemodynamically stable. The fever improved in the first 24 hours. He was immediately started on IV Ganciclovir, which continued for a total of 14 days. His peripheral white count ranged from 6000 to 9900 and his liver enzymes were always normal. Cranial ultrasound was also conducted and revealed no parenchymal abnormalities.

\section{Discussion}

Primary HHV-6 infection manifest in different ways. HHV-6 infection is most often associated with a nonspecific febrile illness occurring between 6 months and 2 years of life. Most common, symptoms include fever, and rash [1]. HHV-6 is most frequently transmitted via the saliva of healthy individuals and enters the body via a mucosal surface. After an incubation period of 10 to 15 days, the illness starts with a prodrome of mild rhinorrhea, sore throat, and conjunctival redness, followed by a high fever [5]. Then, few days later, the fever abates, and the rash of roseola begins as small, erythematous, raised papules on the trunk that spread to the neck and extremities lasting 1 to 3 days [4]. HHV- 6 infection is frequently associated with the first manifestation of benign febrile seizures in childhood. While these seizures are more commonly related to the febrile response to HHV-6 infection, there is a subset of patients who have direct central nervous system HHV-6 infection accounting for the seizures. The frequency of such cases of encephalitis and meningoencephalitis is unclear due to the lack of baseline testing for HHV-6 in children with simple and complex febrile seizures.
Additionally, the exact role that HHV-6 plays in neurological infections is not well understood. Neurologic manifestations of the disease can include generalized, repetitive, and prolonged seizures; as well as ataxia, weakness, hemiplegia, and disturbances in consciousness. Complications such as meningoencephalitis and encephalopathy can be severe and lead to poor outcomes [1].

Conclusive laboratory diagnosis of HHV-6 encephalitis may sometimes be difficult. To investigate whether HHV-6 is an etiological agent of encephalitis, the CSF should be examined for evidence of virus infection by determining the presence of viral sequence using PCR. Elevated serum immunoglobulin M (IgMs) may be suggestive of recent infection and possible post-infectious encephalitis, but also may not be the direct cause of CNS disease. Even positive blood PCRs for HHV-6 does not confirm the presence of this agent in the CNS. To be certain of the diagnosis of active CNS infection with HHV-6, CSF sampling is required, and positive CSF PCRs indicate active CNS infection with HHV-6. While CSF PCR is definitive, serum IgM and PCR can be used to help assure the diagnosis prior to starting toxic therapy [1]. Patients with primary HHV-6 infection are typically mild and usually require only treatment of symptoms such as antipyretics and hydration with no other specific antiviral treatment. Currently there are no approved therapies for the treatment of HHV6 encephalitis [6,7]. Antivirals such as ganciclovir, foscarnet, and cidofovir are usually reserved for immunocompromised patients or those with CNS involvement [4]. These drugs reported success only in a few studies and case reports [6]. Despite the lack of controlled data, foscarnet and ganciclovir (valganciclovir) are recommended as the first line of treatment, with cidofovir as the second line of treatment. The International Herpes Management Forum recommends the use of foscarnet and ganciclovir, either individually or combined, to treat progressive neurologic disease due to HHV-6 infection [6]. Cidofovir, an acyclic nucleoside phosphonate, has been found to be more inhibitory than ganciclovir or foscarnet in vitro [8]. However, it is unclear how well it crosses the blood brain barrier and has associated drug toxicities, so at this time it is not recommended for treating HHV-6 related infections [9]. A high dose of ganciclovir (18mg/ $\mathrm{kg} /$ day) or foscarnet $(60 \mathrm{mg} / \mathrm{kg}$ ) twice a day was suggested for the treatment of a CNS HHV-6 infection [5]. The antiviral medications previously described are effective against active HHV-6 infections, but the indications for treatment, as well as the conditions and duration of drug administration, are not formally approved at this time [9]. New antiviral drugs with a wider range of activity (e.g., brincidofovir) and their combination with immunotherapy (e.g., intravenous immunoglobulin IVIG) or other types of therapy are required to combat roseolovirus-associated CNS complications on a very carefully balanced, individual basis [6]. Numerous pending questions about HHV-6 infection should stimulate future research works on the pathophysiology, diagnosis, and therapy of this virus.

\section{Conclusion}

The degree to which roseolovirus infection contributes to diverse neurological diseases has not been appreciated in the 
past, but such cases must now be fully investigated for these viruses [5]. Improved knowledge of the spectrum of disease and neurologic outcomes for HHV-6 encephalitis will also help to guide antiviral therapy decisions based on clinical severity [1]. The range of CNS manifestations ascribed to these viruses includes asymptomatic infection, febrile convulsions, seizure disorders, meningitis, meningoencephalitis, facial palsy, vestibular neuritis, demyelinating disorders, hemiplegia, and, rarely, fatal encephalitis [6]. If the causative role of HHV-6 in neurological diseases with significant morbidity is not well established, the development of specific treatment for this virus will remain a relatively low priority [8]. For now, it is unlikely that any drug will be developed specifically for the treatment of HHV-6 infections since its severity and morbidity is not well established [8]. In conclusion, HHV-6 may occasionally cause meningitis in young infants, especially in the newborn period. Further studies are required to define the role of HHV-6 and HHV-7 in neurologic disorders such as meningitis or encephalitis in immunocompetent hosts. New studies should focus on finding a safe and effective broad-spectrum antiviral drug and identifying an effective duration of treatment for the appropriate management of roseola associated comorbidities.

\section{Declarations}

1. Ethics approval and consent to participate.

2. The submitted manuscript involves a case report of a human and has been approved by the ethics committee of the Lebanese University.

\section{References}

1. Thrasher AN, Michael J, Chusid, Richard D (2010) HHV-6 infection in a case of an infant with fever, seizures, and shock. Wisconsin Medical Journal 109(3): 153-155.

2. Hukin J, Farrell K, MacWilliam LM, Colbourne M, Waida E, et al. (1998) Case-control study of primary human herpesvirus 6 infection in children with febrile seizures. Pediatrics 101(2): E3.

3. David WK, Richard JW (1998) Human herpesvirus-6: neurologic implications of a newly-described viral pathogen. Journal of Neurovirology 4(5): 474-485.

4. Grady JS (2014) Fifth and sixth diseases: more than a fever and a rash. The Journal of Family Practice 63(10): E1-E5.

5. Ongradi J, Ablashi DV, Yoshikawa T, Stercz B, Ogata M (2017) Roseolovirus associated encephalitis in immunocompetent and immunocompromised individuals. Journal of Neurovirology 23(1): 1-19.

6. Ansari A, Li S, Abzug MJ, Weinberg A (2004) Human herpesviruses 6 and 7 and central nervous system infection in children. Emerging Infectious Diseases Journal 10(8): 1450-1454.

7. Abstract for Reference 116 of Human Herpesvirus 6 infection in children: Clinical manifestation, diagnosis and treatment.

8. Prichard MN, Whitley RJ (2014) The development of new therapies for human herpesvirus 6. Current Opinion in Virology 9: 148-153.

9. Agut H, Bonnafous P, Gautheret DA (2015) Laboratory and clinical aspects of human herpesvirus 6 infection. Clinical Microbiology Reviews 28(2): 313-335.
Creative Commons Attribution 4.0 International License

For possible submissions Click Here

\section{Submit Article}

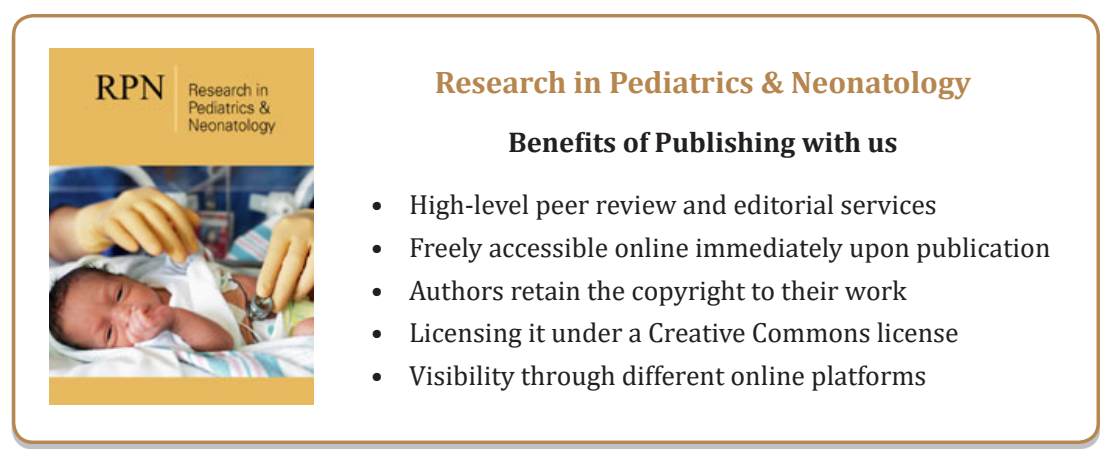

